\title{
Characterization of vitamin D supplementation and clinical outcomes in a large cohort of early Parkinson's disease
}

\author{
Nijee S. Luthra ${ }^{1 *}$, Soeun Kim², Yunxi Zhang ${ }^{2}$, Chadwick W. Christine ${ }^{1,3}$ and On behalf of the NINDS NET-PD
} Investigators

\begin{abstract}
Background: Vitamin D (VitD) deficiency is common in Parkinson's disease (PD) and has been raised as a possible PD risk factor. In the past decade, VitD supplementation for potential prevention of age related conditions has become more common. In this study, we sought to characterize VitD supplementation in early PD and determine as an exploratory analysis whether baseline characteristics or disease progression differed according to reported VitD use.

Methods: We analyzed data from the National Institutes of Health Exploratory Trials in Parkinson's Disease (NET-PD) Long-term study (LS-1), a longitudinal study of 1741 participants. Subjects were divided into following supplement groups according to subject exposure (6 months prior to baseline and during the study): no VitD supplement, multivitamin (MVI), VitD $\geq 400 \mathrm{IU} /$ day, and VitD + multivitamin (VitD+MVI). Clinical status was followed using the Unified Parkinson's Disease Rating Scale, Symbol Digit Modalities Test, total daily levodopa equivalent dose, and Parkinson's Disease Questionnaire.

Results: About 5\% of subjects took VitD alone, 7\% took VitD+MVI, 34\% took MVI alone, while 54\% took no supplement. Clinical outcomes at 3 years were similar across all groups.

Conclusion: This study shows VitD supplementation $\geq 400 \mathrm{IU} /$ day was not common in early PD and that its use was similar to that seen in the US population. At 3 years, there was no difference in disease progression according to vitamin D supplement use.
\end{abstract}

Keywords: 1,25-dihydroxyvitamin D, Parkinson's disease

\section{Background}

Numerous reports have shown that vitamin D (VitD) insufficiency is common in aging, is associated with greater risk of falls and fractures, and that VitD supplementation may reduce the risk of fractures [1]. Because of this observation, and possibly because of numerous studies showing associations of D deficiency with diseases in adults, VitD supplementation has become common. A cross-sectional survey from 2011 to 12 found that about $21 \%$ of adults aged $40-64$ and $38 \%$ of those > 65 take a separate VitD supplement [2].

\footnotetext{
*Correspondence: nijee.luthra@ucsf.edu

${ }^{1}$ Department of Neurology, University of California, 1635 Divisadero, Suite

520-530, San Francisco, CA 94115, USA

Full list of author information is available at the end of the article
}

A relationship of VitD levels and Parkinson's disease (PD) was first recognized in 1997 when Sato and colleagues reported a higher prevalence of VitD deficiency and reduced bone mass in PD [3]. Since then, a number of studies have confirmed these findings [4] and that a mutation in the VitD receptor is associated with an increased risk of developing PD [5]. Studies in experimental animal models of PD have demonstrated neuroprotective effects of VitD [6], lending support to the hypothesis that VitD status may influence PD pathogenesis. The Mini-Finland Health Survey provided additional support of this hypothesis by showing a greater risk for PD in those with lower VitD levels [7].

Because these studies raise the question as to whether VitD supplementation affects PD progression,

(c) The Author(s). 2018 Open Access This article is distributed under the terms of the Creative Commons Attribution 4.0 International License (http://creativecommons.org/licenses/by/4.0/) which permits unrestricted use, distribution, and reproduction in any medium, provided you give appropriate credit to the original author(s) and the source, provide a link to the Creative Commons license, and indicate if changes were made. The Creative Commons Public Domain Dedication waiver (http://creativecommons.org/publicdomain/zero/1.0/) applies to the data made available in this article, unless otherwise stated. 
we performed a study to characterize contemporary VitD supplementation in a large, longitudinal cohort of early PD patients from the National Institutes of Health Exploratory Trials in Parkinson's Disease (NET-PD) Longterm study (LS-1) which enrolled subjects from the United States and Canada from 2007 to 2010. As a an exploratory outcome, we also determined whether baseline characteristics or disease progression outcomes differed according to reported VitD supplement use prior to baseline and during the study observation period.

\section{Methods}

The NET-PD LS-1 study was a large randomized multicenter, double-blind, placebo-controlled study of creatine monohydrate. As previously described, eligible subjects had diagnosis of PD for $<5$ years and had started treatment with levodopa or dopamine agonist for $\geq 90$ days but $<2$ years prior to the baseline visit [8]. Subjects were monitored with annual visits for up to 6 years. The study was halted before completion after it was determined no difference would emerge between the treatment arms [8].

At study entry, subjects were required to report all medications and supplements used in the prior 180 days, although many provided much longer histories of supplement use. We reviewed the concomitant medication records for VitD and multivitamin (MVI) use including supplement brand name (when available), dose, frequency, start date and end date. Of the 1956 records of supplement use (separate records were created for each supplement), 620 (31\%) documented supplement use starting prior to 2005. For those taking specified supplements, the published dose was used. The daily dose was calculated according to dose and frequency. Subjects were stratified according to the following VitD exposure groups: no VitD or MVI supplement (NoSupp), multivitamin where dose of VitD was assumed (if not specified) to be $400 \mathrm{IU} /$ dose (MVI group), $\geq 400 \mathrm{IU} /$ day of VitD (VitD group), or multivitamin plus $\geq$ of $400 \mathrm{IU} /$ day of VitD (VitD+MVI group).

In order to construct defined groups of participants with different dose and longitudinal VitD exposures, subjects were included in the analysis if they had been taking supplements for $\geq 90$ days prior to baseline and continued that supplement for at least 1.5 years of the first 3 years of the study or their records indicated no D supplementation or MVI during this timeframe (NoSupp group). Subjects who took VitD for shorter durations during the study period or at lower doses were excluded.

\section{Assessments}

Because a significant fraction of subjects did not complete the 5-year visit due to early study termination, we calculated mean study outcomes at baseline and the change from baseline in outcomes at 3 years. Outcomes included demographics, Unified Parkinson's Disease Rating Scale (UPDRS) total score and its subscores obtained in the practically defined "On-medication" state, [mental (Part 1 ), activities of daily living (Part 2), and motor (Part 3)], Parkinson's Disease Questionnaire (PDQ39), Scales for Outcomes in Parkinson's Disease-Cognition (SCOPA-COG; only performed at baseline and 5 year visit), Symbol Digit Modalities Test (SDMT), and total daily levodopa equivalent dose (LED).

\section{Statistical analysis}

To investigate baseline characteristics for each group, means and standard deviations were calculated for continuous variables, and percentages were computed for categorical variables. NoSupp, MVI, VitD, VitD+MVI groups were compared using Analysis of variance (ANOVA) test for continuous variables, and Chi-square tests and Fisher's exact tests for categorical variables. Baseline age and age at PD diagnosis was summarized by performing subgroup analyses on males and females, using ANOVA test within each gender group. Pairwise comparisons were assessed using the Hochberg's multiple testing method. Changes from baseline in the outcomes were computed at the third year visit, and mean changes were compared for four groups using ANOVA test. The ANOVA test was used to compare mean age at diagnosis for the four supplement groups, within each age categories. In order to adjust for baseline morbidity, we also performed the outcome analysis using mixed models, adjusting for baseline age and the corresponding baseline score as fixed effects and including site as a random effect. SAS 9.4 was used for the analyses.

\section{Results}

Of the 1741 subjects who enrolled in the study, 943 subjects had been taking MVI, VitD, or VitD+MVI for $\geq 90$ days prior to baseline and at least 1.5 years of duration of the study or reported no supplement use (NoSupp group) during this timeframe. Of these subjects, 54\% of subjects took NoSupp, 34\% took MVI, 5\% took VitD, and 7\% took VitD+MVI (where for VitD, the dose was $>400$ IU/day).

Although disease severity as measured by rating scales was similar between the groups at baseline, the age at baseline, and the related age at diagnosis of PD, was higher in subjects taking supplements [ MVI $(60.2 \pm 8.7)$, $\operatorname{VitD}(63.9 \pm 6.6)$ and VitD+MVI $(64.1 \pm 7.7)]$ compared to subjects in NoSupp $(58.5 \pm 9.7)$ group (Table 1). In order to address whether supplement use differed according to age, we then performed an analysis segregated according to age range $(<50,50-59,60-69, \geq 70)$ at $\mathrm{PD}$ diagnosis (Table 2). This analysis confirmed that VitD use was higher in older patients and that when sorted by age range, the mean ages at diagnosis were not different. Time 
Table 1 Baseline Characteristics

\begin{tabular}{|c|c|c|c|c|c|}
\hline & $\begin{array}{l}\text { No } \\
\text { Supplement } \\
(N=511)\end{array}$ & $\begin{array}{l}\text { MVI } \\
(N=322)\end{array}$ & $\begin{array}{l}D \\
(N=42)\end{array}$ & $\begin{array}{l}D+M V I \\
(N=68)\end{array}$ & $P$ value \\
\hline & Mean (SD) & & & & F test \\
\hline Age in years & $60.1(9.7)$ & $61.8(8.6)^{*}$ & $65.3(6.4)^{*}$ & $65.5(7.7)^{*}$ & $<0.0001$ \\
\hline Age at PD Dx & $58.5(9.7)$ & $60.2(8.7)^{*}$ & $63.9(6.6)^{*}$ & $64.1(7.7)^{*}$ & $<0.0001$ \\
\hline Yrs since Dx & $1.6(1.1)$ & $1.7(1.2)$ & $1.3(1.0)$ & $1.4(0.8)$ & 0.07 \\
\hline Years since Dx to D-treatment & $1.1(1.1)$ & $1.3(1.2)$ & $0.9(1.0)$ & $1.0(0.8)$ & 0.08 \\
\hline UPDRS total & $25.1(10.3)$ & $25.4(10.3)$ & $25.1(11.4)$ & $24.5(9.8)$ & 0.92 \\
\hline UPDRS mental & $1.2(1.3)$ & $1.2(1.3)$ & $1.4(1.4)$ & $1.2(1.3)$ & 0.85 \\
\hline UPDRS ADL & $6.8(3.7)$ & $6.8(3.6)$ & $6.2(3.9)$ & $6.4(3.4)$ & 0.66 \\
\hline PDQ 39 summary & $12.9(10.4)$ & $11.5(8.7)$ & $12.5(9.6)$ & $10.5(8.3)$ & 0.08 \\
\hline SCOPA COG & $30.4(5.1)$ & $30.4(5.2)$ & $30.9(4.6)$ & $31.1(5.0)$ & 0.71 \\
\hline SDMT & $45.1(11.1)$ & $44.4(10.9)$ & $45.2(11.4)$ & $45.9(11.4)$ & 0.71 \\
\hline \multirow[t]{2}{*}{ Total daily LED } & $374.9(224.9)$ & $371.3(232.8)$ & $390.0(208.0)$ & $367.4(355.4)$ & 0.96 \\
\hline & Frequency (\%) & & & & $X^{2}$ test $^{a}$ \\
\hline Female \% & $144(28.2 \%)$ & $94(29.2 \%)$ & $27(64.3 \%)$ & 38 (55.9\%) & $<0.0001$ \\
\hline Non-Hispanic white & $448(87.7 \%)$ & $303(94.1 \%)$ & 41 (97.6\%) & 66 (97.1\%) & 0.001 \\
\hline Education $>17$ years $^{\mathrm{a}}$ & 149 (29.2\%) & 118 (36.7\%) & 16 (38.1\%) & 18 (26.5\%) & 0.08 \\
\hline
\end{tabular}

Abbreviations: PD Parkinson's disease, Dx diagnosis, yrs years, D-treatment dopaminergic treatment (dopamine agonist or levodopa), UPDRS Unified Parkinson's Disease Rating Scale, ADL activities of daily living, PDQ Parkinson's disease questionnaire, SCOPA COG Scales for outcomes of Parkinson's disease-Cognition, SDMT symbol digit modalities test, LED levodopa equivalent dose

${ }^{*} p<.05$ compared to NoSupp, according to pairwise comparisons Hochberg method

${ }^{a} X^{2}$ test was calculated by Fisher's exact test

from diagnosis to start of dopaminergic treatment was not different in subjects taking supplements (Table 1). The percentage of females and non-Hispanic whites was higher in the VitD and VitD+MVI groups.

No differences in mean clinical outcomes were noted between groups at 3 years (Table 3). An analysis using mixed models, adjusted for baseline age and corresponding baseline scores as covariates, also failed to show a difference in outcomes according to supplement use (Table 4).

\section{Discussion}

In this analysis of the NET-PD LS1 cohort, we found that about $12 \%$ of subjects took $>400 \mathrm{IU} /$ day of VitD supplementation while $34 \%$ took a MVI estimated to contain $400 \mathrm{IU} /$ day of VitD. Disease progression as measured by a variety of rating scales at 3 years was similar in all 4 groups. Although methods of ascertainment were quite different, these rates of MVI and VitD use are in line with rates reported in the United States population from the large cross-sectional NHANES study of 34\% for MVI use and $11 \%$ for separate VitD supplement use from 2007 to 8 [2].

Our finding that the age at diagnosis of PD was older in the MVI group, VitD and VitD plus MVI groups could suggest a disease delaying effect. However, further analysis of this data segregated according to age range of onset showed that VitD supplementation was higher in

Table 2 Analysis of Supplement Use by Age Range

\begin{tabular}{|c|c|c|c|c|c|c|}
\hline Age at Diagnosis & Statistics & No Supp $(N=511)$ & MVI $(N=322)$ & VitD $(N=42)$ & VitD + MVI $(N=68)$ & $p$-value \\
\hline \multirow[t]{2}{*}{$<50$} & Freq (\%) & 91 (70\%) & $35(27 \%)$ & $1(1 \%)$ & $2(2 \%)$ & \\
\hline & Mean (SD) & $44.04(4.54)$ & $44.63(5.07)$ & 45.00 (NA) & $46.50(3.54)$ & 0.83 \\
\hline \multirow[t]{2}{*}{$50-59$} & Freq (\%) & $182(58 \%)$ & 106 (34\%) & $10(3 \%)$ & $17(5 \%)$ & \\
\hline & Mean (SD) & $54.85(2.73)$ & $54.87(2.96)$ & $56.70(3.09)$ & $56.06(2.88)$ & 0.08 \\
\hline \multirow[t]{2}{*}{ 60-69 } & Freq (\%) & $164(46 \%)$ & 139 (39\%) & $23(6 \%)$ & $30(9 \%)$ & \\
\hline & Mean (SD) & $63.81(2.90)$ & $64.12(2.85)$ & $64.83(3.16)$ & $64.13(2.76)$ & 0.42 \\
\hline \multirow[t]{2}{*}{$\geq 70$} & Freq (\%) & 74 (52\%) & 42 (29\%) & $8(6 \%)$ & 19 (13\%) & \\
\hline & Mean (SD) & $73.54(3.21)$ & 73.29 (2.62) & $72.38(1.06)$ & 73.37 (3.92) & 0.78 \\
\hline
\end{tabular}


Table 3 Mean Change in Outcomes and at 3-year Visit

\begin{tabular}{|c|c|c|c|c|c|}
\hline & No D Supplement $(N=511)$ & MVI $(N=322)$ & $D(N=42)$ & $D+M V I(N=68)$ & \multirow{2}{*}{$\begin{array}{l}P \text { value } \\
F \text { test }\end{array}$} \\
\hline & \multicolumn{4}{|l|}{ Mean (SD) } & \\
\hline Change in UPDRS total & $5.5(11.8)$ & $6.2(12.0)$ & $4.9(9.8)$ & $9.2(14.3)$ & 0.12 \\
\hline Change in UPDRS mental & $0.5(1.5)$ & $0.5(1.5)$ & $0.6(2.0)$ & $0.6(1.8)$ & 0.83 \\
\hline Change in UPDRS ADL & $2.0(4.4)$ & $2.3(4.5)$ & $1.9(3.6)$ & $3.0(4.7)$ & 0.30 \\
\hline Change in PDQ 39 summary & $4.6(10.4)$ & $4.3(9.6)$ & $4.0(11.3)$ & $4.8(10.5)$ & 0.96 \\
\hline Change in SDMT & $0.002(9.5)$ & $-0.2(9.4)$ & $-1.6(11.0)$ & $-0.8(13.3)$ & 0.73 \\
\hline Change in Total daily LED & $294.6(367.1)$ & $268.4(339.8)$ & $220.6(244.5)$ & $271.5(346.4)$ & 0.48 \\
\hline
\end{tabular}

Abbreviations: UPDRS Unified Parkinson's Disease Rating Scale, ADL activities of daily living, PDQ Parkinson's disease questionnaire, SDMT symbol digit modalities test, $L E D$ levodopa equivalent dose

older participants (suggesting reverse causation), and that mean age at diagnosis was no longer significantly different between groups.

Our finding that VitD supplementation did not affect PD progression is consistent with observations from the

Table 4 Change in Outcomes at 3-year Visit according to mixed models $^{a}$

\begin{tabular}{|c|c|c|c|}
\hline Outcome & Group & Estimate & $P$ value \\
\hline \multirow[t]{4}{*}{ Change in UPDRS total } & No Supplement & . & \\
\hline & $\mathrm{MVI}$ & 0.248 & 0.77 \\
\hline & $\mathrm{D}$ & -1.517 & 0.43 \\
\hline & $\mathrm{D}+\mathrm{MVl}$ & 2.540 & 0.10 \\
\hline \multirow[t]{4}{*}{ Change in UPDRS ADL } & No Supplement & . & . \\
\hline & $\mathrm{MVI}$ & 0.117 & 0.70 \\
\hline & $\mathrm{D}$ & -0.555 & 0.43 \\
\hline & $\mathrm{D}+\mathrm{MVl}$ & 0.469 & 0.41 \\
\hline \multirow[t]{4}{*}{ Change in UPDRS mental } & No Supplement & . & \\
\hline & $\mathrm{MVI}$ & -0.058 & 0.58 \\
\hline & $\mathrm{D}$ & 0.083 & 0.73 \\
\hline & $\mathrm{D}+\mathrm{MVI}$ & 0.083 & 0.67 \\
\hline \multirow[t]{4}{*}{ Change in PDQ39 } & No Supplement & . & . \\
\hline & $\mathrm{MVI}$ & -0.571 & 0.43 \\
\hline & $\mathrm{D}$ & -0.986 & 0.55 \\
\hline & $\mathrm{D}+\mathrm{MVI}$ & -0.422 & 0.75 \\
\hline \multirow[t]{4}{*}{ Change in SDMT } & No Supplement & . & \\
\hline & MVI & -0.041 & 0.95 \\
\hline & $\mathrm{D}$ & -0.654 & 0.67 \\
\hline & $\mathrm{D}+\mathrm{MVl}$ & 0.871 & 0.48 \\
\hline \multirow[t]{4}{*}{ Change in Total daily LED } & No Supplement & . & \\
\hline & $\mathrm{MVI}$ & -23.174 & 0.34 \\
\hline & $\mathrm{D}$ & -63.506 & 0.25 \\
\hline & $\mathrm{D}+\mathrm{MVl}$ & -19.346 & 0.66 \\
\hline
\end{tabular}

Abbreviations: UPDRS Unified Parkinson's Disease Rating Scale, ADL activities of daily living, $P D Q$ Parkinson's disease questionnaire, SDMT symbol digit modalities test, LED levodopa equivalent dose

${ }^{a}$ Model controlled for baseline age and the corresponding baseline score as fixed effects and including site as a random effect
DATATOP study of early PD in which no correlation was seen between VitD levels and disease progression [9]. However, a more recent 3-year study did find a small, but significant association between VitD levels measured at baseline and motor progression [10], raising the possibility that the amount of VitD supplementation by the NET-PD LS1 participants was inadequate to provide benefit.

Although there is evidence of high prevalence of VitD deficiency in early PD [9] and established PD [11], there is currently little published regarding the effect of VitD supplementation in PD. Suzuki et al. conducted a randomized, placebo-controlled study examining the effect supplementation with $1200 \mathrm{IU} /$ day of vitamin D3/d for 12 months [12]. They found that D supplementation was associated with smaller changes in a number of outcomes measures including the Hoehn and Yahr stage, UPDRS part II and total, and some domains of the PDQ39 as well as the participants' VitD receptor genotype. Their study differed from our study in a number of ways since it was randomized, larger doses of VitD were specified, and because subjects started treatment after baseline while our study followed subjects on established VitD supplementation for a longer duration of time. Their design examines both potential treatment and neuroprotective effects of $\mathrm{D}$ supplementation while our study more specifically examined baseline characteristics of supplemented patients and their progression.

Our study has several limitations. As it is a secondary analysis, subjects were not randomized to treatment. Also, subjects were analyzed according to reported supplement dose with no independent measurements of serum VitD levels nor were we able to account for differing levels of sunlight exposure. Moreover although many subjects provided information regarding supplement use for years prior to study entry, study procedures only required report of supplement use in the 180 days prior to baseline, which for the majority of subjects was after their PD diagnosis. There were also lower numbers of subjects taking VitD or VitD+MVI compared to the other groups. Finally, while this study did not show differences in disease progression in the first three years 
after starting dopaminergic treatment, it is possible that D supplementation could affect later PD progression.

\section{Conclusion}

Although VitD insufficiency is common in PD, this study shows VitD > $400 \mathrm{IU} /$ day supplementation was not common and was similar to the rate observed in cross-sectional studies of the general US population according to age group. Although no difference in early disease progression was observed in patients taking VitD supplementation, it remains possible that consistent VitD supplementation could reduce the development of disability related to bone health, since osteoporosis is more common in PD and the risk of falls and bone fracture increase with disease duration. These data may be helpful in the design of studies testing the effect of vitamin D supplementation over a longer time frame.

\begin{abstract}
Abbreviations
ANOVA: Analysis of variance; LED: Levodopa equivalent dose; LS-1: Longterm study; MVI: Multivitamin; NET-PD: National Institutes of Health Exploratory Trials in Parkinson's Disease; NoSupp: No supplement; PD: Parkinson's disease; PDQ39: Parkinson's Disease Questionnaire; SCOPA-COG: Scales for Outcomes in Parkinson's Disease-Cognition; SDMT: Symbol Digit Modalities Test; UPDRS: Unified Parkinson's Disease Rating Scale; Vitamin D: VitD
\end{abstract}

\section{Acknowledgements}

We gratefully acknowledge patients and their families for participation in the NET-PD LS-1 clinical trial.

\section{Funding}

The NET-PD LS-1 trial received funding support from the National Institute for Neurological Disorders and Stroke (U01NS43128 and U01 NS043127). For the current report, a secondary analysis of trial data, the authors received no specific funding to support this work.

\section{Availability of data and materials}

The data that support the findings of this study are available from the NETPD investigators but restrictions apply to the availability of these data, which were used under license for the current study, and so are not publicly available. Data are however available from the authors upon reasonable request and with permission of the NET-PD investigators.

\section{Authors' contributions}

$\mathrm{NL}$ and CC made substantial contributions to conception, design, analysis and interpretation of data, drafting the manuscript and gave final approval of the version to be published. SK and YZ made substantial contributions to acquisition of data, statistical analysis and interpretation of data. Each author has participated sufficiently in the work to take public responsibility for appropriate portions of the content and agreed to be accountable for all aspects of the work in ensuring that questions related to the accuracy or integrity of any part of the work are appropriately investigated and resolved. All authors read and approved the final manuscript.

\section{Ethics approval and consent to participate}

The NET-PD LS-1 trial was registered at wWW.clinicaltrials.gov (NCT00449865), Study procedures were reviewed and approved by the institutional review board for each site, and was carried out in accordance with the ethical standards and regulations of each site. Prior to enrollment of participants, each provided written informed consent to study participation.

\section{Consent for publication}

Not applicable, as the manuscript does not contain any individual person's data.

\section{Competing interests}

The authors declare that they have no competing interests.

\section{Publisher's Note}

Springer Nature remains neutral with regard to jurisdictional claims in published maps and institutional affiliations.

\section{Author details}

${ }^{1}$ Department of Neurology, University of California, 1635 Divisadero, Suite 520-530, San Francisco, CA 94115, USA. ²Department of Biostatistics and Data Science, University of Texas Health Center, 1200 Pressler Street, Houston, TX 77030, USA. ${ }^{3}$ Department of Neurology, University of California San Francisco, 400 Parnassus Ave Box 0348, San Francisco, CA 94122, USA.

Received: 8 August 2018 Accepted: 11 October 2018

Published online: 31 October 2018

References

1. Rosen CJ. Clinical practice Vitamin D insufficiency. N Engl J Med. 2011. https://doi.org/10.1056/NEJMcp1009570.

2. Kantor ED, Rehm CD, Du M, White E, Giovannucci EL. Trends in dietary supplement use among US adults from 1999-2012. JAMA. 2016. https://doi. org/10.1001/jama.2016.14403.

3. Sato Y, Kikuyama M, Oizumi K. High prevalence of vitamin D deficiency and reduced bone mass in Parkinson's disease. Neurology. 1997. https://doi.org/ 10.1212/WNL.49.5.1273.

4. Wang L, Evatt ML, Maldonado LG, Perry WR, Ritchie JC, Beecham GW, et al. Vitamin D from different sources is inversely associated with Parkinson disease. Mov Disord. 2015. https://doi.org/10.1002/mds.26117.

5. Han $X, X$ ue $L, L i Y$, Chen $B$, Xie A. Vitamin D receptor gene polymorphism and its association with Parkinson's disease in Chinese Han population. Neurosci Lett. 2012. https://doi.org/10.1016/j.neulet.2012.07.033.

6. Deluca GC, Kimball SM, Kolasinski J, Ramagopalan SV, Ebers GC. Review: the role of vitamin D in nervous system health and disease. Neuropathol Appl. 2013. https://doi.org/10.1111/nan.12020

7. Knekt P, Kilkkinen A, Rissanen H, Marniemi J, Sääksjärvi K, Heliövaara M. Serum vitamin D and the risk of Parkinson disease. Arch Neurol. 2010. https://doi.org/10.1001/archneurol.2010.120.

8. Kieburtz K, Tilley BC, Elm JJ, Babcock D, Hauser R, Ross GW, Augustine AH, et al. Effect of Creatine monohydrate on clinical progression in patients with Parkinson disease. JAMA. 2015. https://doi.org/10.1001/jama.2015.120.

9. Evatt ML, DeLong MR, Kumari M, Auinger P, McDermott MP, Tangpricha V. High prevalence of hypovitaminosis D status in patients with early Parkinson disease. Arch Neurol. 2011. https://doi.org/10.1001/archneurol.2011.30.

10. Sleeman I, Aspray T, Lawson R, Coleman S, Duncan G, Khoo TK. The role of vitamin D in disease progression in early Parkinson's disease. J Parkinsons Dis. 2017. https://doi.org/10.3233/JPD-171122.

11. Wang L, Evatt ML, Maldonado LG, Perry WR, Ritchie JC, Beecham GW, et al. Vitamin D from different sources is inversely associated with Parkinson disease. Mov Disord. 2014. https://doi.org/10.1002/mds.26117.

12. Suzuki M, Yoshioka M, Hashimoto M, Murakami M, Noya M, Takahashi D, et al. Randomized, double-blind, placebo-controlled trial of vitamin D supplementation in Parkinson disease. Am J Clin Nutr. 2013. https://doi.org/10. 3945/ajen.112.051664.

Ready to submit your research? Choose BMC and benefit from:

- fast, convenient online submission

- thorough peer review by experienced researchers in your field

- rapid publication on acceptance

- support for research data, including large and complex data types

- gold Open Access which fosters wider collaboration and increased citations

- maximum visibility for your research: over $100 \mathrm{M}$ website views per year

At BMC, research is always in progress.

Learn more biomedcentral.com/submission 